\title{
Research on Track Deception Technology Based on Jamming of Flexible Lines by Jammers
}

\author{
SHI Zhouyan ${ }^{1, \mathrm{a}}$, WANG Min ${ }^{1, \mathrm{a}}$, ZHANG Lu ${ }^{1, \mathrm{a}}$, MA Linzhi ${ }^{1, \mathrm{a}}$ \\ ${ }^{1}$ Air Force Early Warning Academy, Wuhan 430019, China;
}

\begin{abstract}
In order to effectively interfere with radar networks, this paper combines the principle of distance deception jamming and the traditional track deception jamming model, a track deception jamming model based on jamming aircraft flexible route penetration was established. The relevant motion parameter solving method flow is given and simulated. The results show that the model not only can achieve track deception, but also can effectively increase the degree of freedom of the jammers' invasion route, making the penetration route of the jammers more flexible and controllable than the traditional.
\end{abstract}

\section{Introduction}

In recent years, radar network technology has achieved rapid development. How to effectively interfere with the radar network and protect its own target has become an urgent problem to be solved. Among various types of interference, track deception and interference technology has the advantages of less resources and high interference utilization. Therefore, this technology has become a research hotspot in the field of electronic interference.

Reference [1] describes the principle of track deception; Reference [2] establishes a multi-aircraft coordinated track deception optimal control model; Reference [3] proposes a radar network track deception technique based on centralized track planning and distributed track coordination; Reference [4] proposed a mathematical model to optimize the interference track; Reference [5] simplifies the model of track deception jamming in drones; Reference [6] proposes a compensation technique. The above studies have achieved good results.

Above the researches, only consider how to generate a false track. Track fraud is mainly achieved through distance deception jamming technology[7]. In the case of false track determination, the movement of the jammers are constrained by false targets. In the study, the jammers' penetration routes are generally made parallel to the false track, which reduces the degree of freedom of the attack route from $3 \mathrm{D}$ to $1 \mathrm{D}[2]$. To a great extent, it limits the movement of the jammers. However, in practice, when a multi-penetration radar network based composite interference press and deception[8-9], when the jammer formations are underway, it is sometimes necessary to avoid the radar to detect the exposed area or to adjust the penetration route to suppress the interference. This article presents a jammer flexible penetration of deception track model, the degree of freedom jammer route extended to two-dimensional, and the motion characteristics of the jammers are described by the parameter.

\section{Basic Principles}

Distance spoofing is the basis of track deception. The jammers intercept the radar pulse signal and forward it after the delay. The main lobe enters the radar receiver, thus producing realistic false targets on the radar. By setting the forwarding delay and controlling the distance between the false target and the radar, a false track can be generated on the radar. In the case of radar network penetration, false trajectories need to be deceived by jamming machines to interfere with all radars, so that false trajectories generated on different radars coincide in position, thereby realizing deception of radar tracks. Track deception diagram is shown in Figure 1.

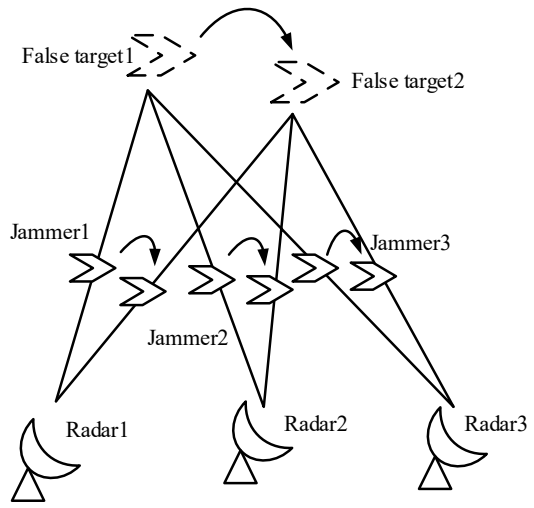

Fig.1. Track deception 
From Figure 1, False radar track curves formed with a curved surface, with the premise to the environment and allow the jammers' performance, only these jammers held on the surface. Therefore, this feature can be used to flexibly control the jammers route.

In order to implement the jammers' flexible route penetration, the following conditions must be met:

1) Each jammer flexibility to adjust the velocity of flight, azimuth and elevation, to ensure the radar in connection with false targets.

2) The jammer has Digital RF memory (DRFM), which can receive and delay the radar signal. This technology requires a high delay and therefore requires precise control of the delay time.

3) The location and number of radars are known to ensure that a jammer interferes with a radar.

4) The penetration line setting of the jammer needs to be performed with equipment performance and environment permitting.

\section{Flexible Route Defense Mathematical Model}

\subsection{Sports Model}

When there is only one-dimensional degree of freedom, and the jammer course is parallel to the false track, the description of the motion characteristics only needs to calculate the velocity and heading angle. In the process of flexible route penetration, it is necessary to describe its motion characteristics through velocity, velocity azimuth and velocity pitch angle, as shown in Figure 2.

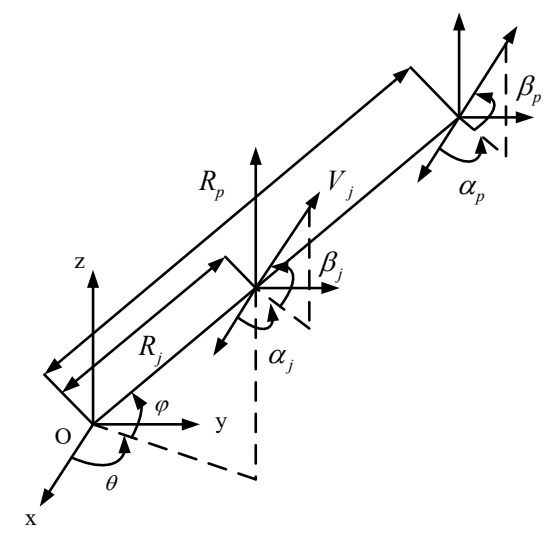

Fig. 2. Motion model

Among them, O, I, $\mathrm{P}$ represent radar, jammer and false target; $\mathrm{R}$ represents distance; $\mathrm{V}$ represents velocity; $\theta$ represents position azimuth; ${ }^{\varphi}$ represents position pitch angle; $\alpha$ represents velocity azimuth; $\beta$ represents velocity pitch angle. In the rectangular coordinate system, the equation of motion of jammer and false target can be expressed, as shown in formula (1).

$$
\left\{\begin{array}{l}
\dot{x_{i}}=V_{i} \cos \alpha_{i} \cos \beta_{i} \\
\dot{y_{i}}=V_{i} \sin \alpha_{i} \cos \beta_{i} \\
\dot{z_{i}}=V_{i} \sin \beta_{i}
\end{array}\right.
$$

Among them, $i=\left\{\begin{array}{l}p, \text { False target } \\ j, j \text {-th jammer }\end{array} ; \dot{\otimes}\right.$ means

' $\otimes$ derivative of time'. To facilitate the description of the relationship between radar, jammer and false target, the motion model can be converted to a spherical coordinate system with the origin of the radar through the relationship between the spatial Cartesian coordinate system and the spherical coordinate system. In the form of a matrix can be expressed, as shown in formula (2).

$$
\left[\begin{array}{c}
\dot{R}_{i} \\
R_{i} \dot{\theta} \cos \varphi \\
\dot{R_{i}} \dot{\varphi}
\end{array}\right]=\left[\begin{array}{ccc}
\cos \varphi \cos \theta & \cos \varphi \sin \theta & \sin \varphi \\
-\sin \theta & \cos \theta & 0 \\
-\sin \varphi \cos \theta & -\sin \varphi \sin \theta & \cos \varphi
\end{array}\right]\left[\begin{array}{c}
\dot{x}_{i} \\
\dot{y_{i}} \\
\dot{z_{i}}
\end{array}\right]
$$

Simultaneous formula (1) (2):

$$
\begin{aligned}
& \dot{R}_{i}=V_{i}\left(\cos \varphi \cos \theta \cos \alpha_{i} \cos \beta_{i}+\right. \\
& \left.\cos \varphi \sin \theta \sin \alpha_{i} \cos \beta_{i}+\sin \varphi \sin \beta_{i}\right) \\
& \dot{\theta}=\frac{V_{i}}{R \cos \varphi}\left(-\sin \theta \cos \alpha_{i} \cos \beta_{i}+\right. \\
& \left.\cos \theta \sin \alpha_{i} \cos \beta_{i}\right) \\
& \dot{\varphi}=\frac{V_{i}}{R}\left(-\sin \varphi \cos \theta \cos \alpha_{i} \cos \beta_{i}-\right. \\
& \left.\sin \varphi \sin \theta \sin \alpha_{i} \cos \beta_{i}+\cos \varphi \sin \beta_{i}\right)
\end{aligned}
$$

Simultaneous formula (3) (4) (5) can obtain velocity, velocity azimuth and velocity pitch angle, as shown in formula (6) (8).

$$
\begin{gathered}
V_{i}=\left(\left(\dot{R}_{i}\right)^{2}+\left(R_{i} \dot{\theta} \cos \varphi\right)^{2}+\left(R_{i} \dot{\varphi}\right)^{2}\right)^{1 / 2} \\
\alpha_{i}=\arctan (P / Q) \\
\beta_{i}=\arcsin \left(\frac{\dot{R}_{i}}{V_{i}}-P \cos \varphi \cos \theta-Q \cos \varphi \sin \theta\right)
\end{gathered}
$$

In the above formulas,

$$
\left\{\begin{array}{l}
P=\frac{1}{V_{i}}\left(\dot{\theta} R_{i} \cos \varphi \cos \theta+\dot{R}_{i} \cos \varphi \sin \theta-\dot{\varphi} R_{i} \sin \varphi \sin \theta\right) \\
Q=\frac{1}{V_{i}}\left(-\dot{\theta} R_{i} \cos \varphi \sin \theta+\dot{R}_{i} \cos \varphi \cos \theta-\dot{\varphi} R_{i} \sin \varphi \cos \theta\right)
\end{array} .\right.
$$

From the above model, we need to calculate six coupling quantities to calculate equations (6) (8), coupling quantities are: $R 、 \theta 、 \varphi 、 \dot{R}$ 、 $\dot{\theta}$ and $\dot{\varphi}$.From the geometric relationship of Figure 2, we can get the formula(9). 


$$
\left\{\begin{array}{l}
R_{i}=\sqrt{\left(x_{i}-x_{r}\right)^{2}+\left(y_{i}-y_{r}\right)^{2}+\left(z_{i}-z_{r}\right)^{2}} \\
\theta=\arctan \left[\left(y_{i}-y_{r}\right) /\left(x_{i}-x_{r}\right)\right] \\
\varphi=\arcsin \left[\left(z_{i}-z_{r}\right) / r_{i}\right] \\
\dot{R}_{i}=\left[\left(x_{i}-x_{r}\right) \dot{x}_{i}+\left(y_{i}-y_{r}\right) \dot{y}_{i}+\left(z_{i}-z_{r}\right) \dot{z}_{i}\right] / R_{i} \\
\dot{\theta}=\frac{\left(x_{i}-x_{r}\right) \dot{y}_{i}-\left(y_{i}-y_{r}\right) \dot{x}_{i}}{\sqrt{\left(x_{i}-x_{r}\right)^{2}+\left(y_{i}-y_{r}\right)^{2}}} \\
\dot{\varphi}=\frac{\dot{z_{i}} \sqrt{\left(x_{i}-x_{r}\right)^{2}+\left(y_{i}-y_{r}\right)^{2}}}{r_{i}^{2}}- \\
\frac{\left(z_{i}-z_{r}\right)}{r_{i}^{2}} \frac{\left(x_{i}-x_{r}\right) \dot{x}_{i}+\left(y_{i}-y_{r}\right) \dot{y_{i}}}{\sqrt{\left(x_{i}-x_{r}\right)^{2}+\left(y_{i}-y_{r}\right)^{2}}}
\end{array}\right.
$$

In summary, Jammer's movement status can be described by the formula (6) to (8).

\subsection{Position Model}

Interference by the establishment of the position of the model geometric relationships, coordinate jammers can be obtained. The coordinates of radar,jammers and false targets, with the formula (9) a coupling amount can be determined six, additional delay can be obtained from jamming, and then find the jammer to signal the delay amount is obtained.

To perform distance deception jamming on the radar, it is necessary to guarantee the jammer on the connection between the radar and the false target. The line between radar $\left(x_{r}, y_{r}, z_{r}\right)$ and false targets $\left(x_{p}, y_{p}, z_{p}\right)$ can be expressed, as shown in formula (10).

$$
\frac{x-x_{p}}{x_{r}-x_{p}}=\frac{y-y_{p}}{y_{r}-y_{p}}=\frac{z-z_{p}}{z_{r}-z_{p}}
$$

Start with the two-dimensional top view of the confrontation scenario, as shown in Figure 3. The route of the jammers can be set in a two-dimensional top view as required. Set the k-th jammer on the two-dimensional plane as $y=f_{k}(x)$.

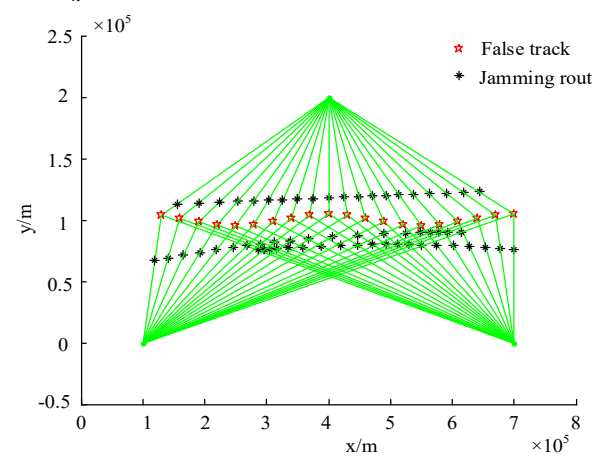

Fig.3.Interference 2D

Extend Figure 3 to three-dimensional space, At this point, $y=f_{k}(x)$ is represented as a surface perpendicular to the ground. The position of the jammers in the three-dimensional space is the intersection of the straight line and the curved surface $y=f_{k}(x)$ where the radar false target is located, as shown in Figure 4.

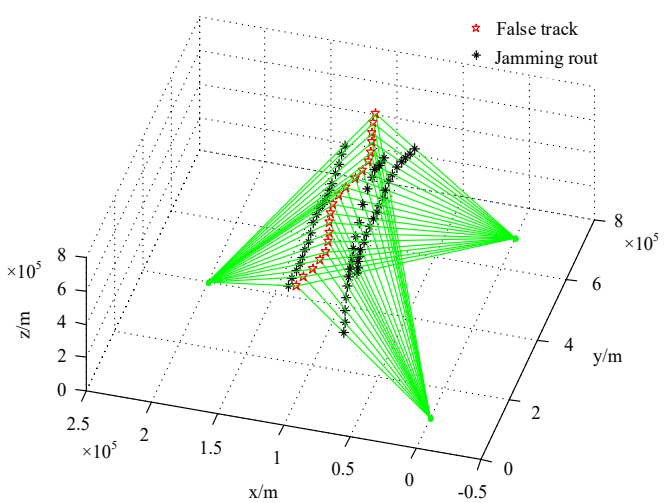

Fig.4.Interference 3D

Bring $y=f_{k}(x)$ into formula (10), the position of the jammers can be obtained, as shown in formula (11).

$$
\left\{\begin{array}{l}
x_{i}=\left(f_{k}\left(x_{i}\right)-y_{p}\right)\left(x_{r}-x_{p}\right) /\left(y_{r}-y_{p}\right)+x_{p} \\
y_{i}=f_{k}\left(x_{i}\right) \\
z_{i}=\left(f_{k}\left(x_{i}\right)-z_{p}\right)\left(z_{r}-z_{p}\right) /\left(y_{r}-y_{p}\right)+x_{p}
\end{array}\right.
$$

Finally, $R_{p}$ and $R_{j}$ are obtained from the coordinates of the radar, the jammers, and the dummy target, and the delay amount of the jammers to the signal can be obtained, as shown in formula (12).

$$
\Delta t= \begin{cases}2\left(R_{p}-R_{j}\right) / c & R_{p}<R_{j} \\ T_{r}+2\left(R_{p}-R_{j}\right) / c & R_{p}>R_{j}\end{cases}
$$

In summary, with $\mathrm{N}$ jammers, the solution steps for the flexible route penetration model as shown in Figure 5.

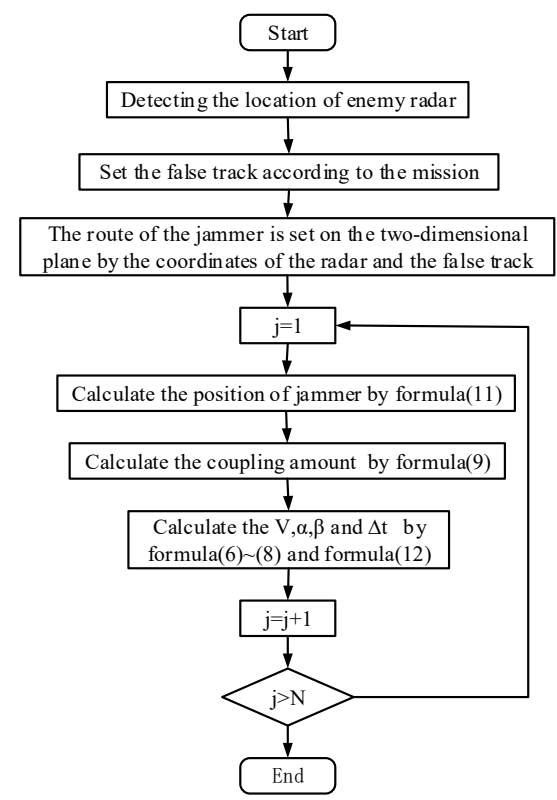

Fig.5.The model solution flowchart

\section{Simulation Analysis}

First of all, suppose the coordinates of the three radars of the enemy are $\left(1 \times 10^{5}, 0,0\right),\left(4 \times 10^{5}, 2 \times 10^{5}, 0\right),\left(7 \times 10^{5}, 0,0\right)$ (Unit: $\left.\mathrm{m}\right)$. Track jamming on the radar network by three jammers, 
the flight time is 2000s. The starting point for planning a false track is $\left(1 \times 10^{5}, 1 \times 10^{5}, 0\right)$. The track expression as shown in formula (13).

$$
\left\{\begin{array}{l}
x=1 \times 10^{5}+300 t \\
y=1 \times 10^{5}+5000 \cos (0.002 \pi t) \\
z=6000
\end{array}\right.
$$

The assumed operational needs, set the surface expression perpendicular to the ground where the jammer is located to be formula (14).

$$
\left\{\begin{array}{l}
y_{1}=-10^{-7}\left(x-6 \times 10^{5}\right)^{2}+9 \times 10^{4} \\
y_{2}=0.02 x+1.1 \times 10^{5} \\
y_{3}=-10^{-7}\left(x-5 \times 10^{5}\right)^{2}+8 \times 10^{4}
\end{array}\right.
$$

Substituting formula (13) (14) into the model of Figure5,three jammers' velocity, velocity azimuth, velocity pitch angle and delay amount can be obtained, as shown in Figure6 Figure9.

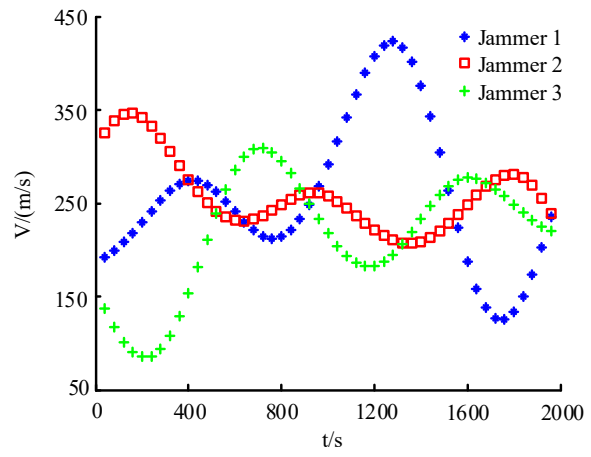

Fig. 6.Jammers' Velocity

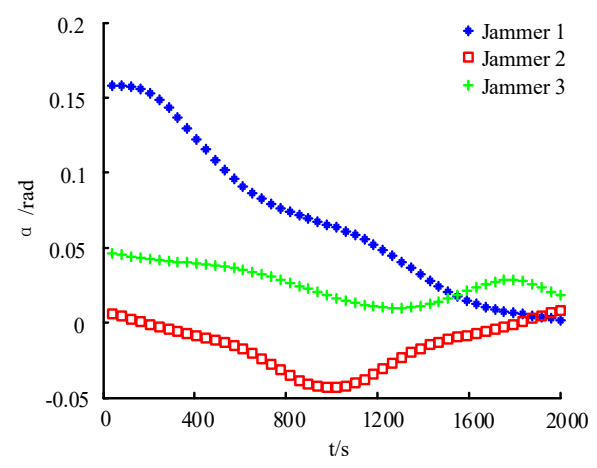

Fig.7.Jammers' Velocity Azimuth

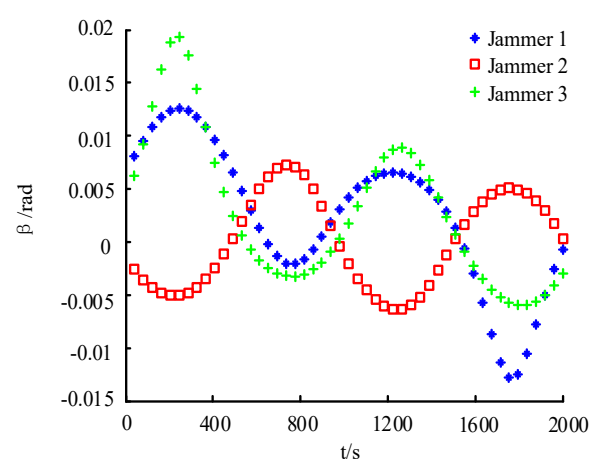

Fig.8.Jammers' Velocity Pitch Angle

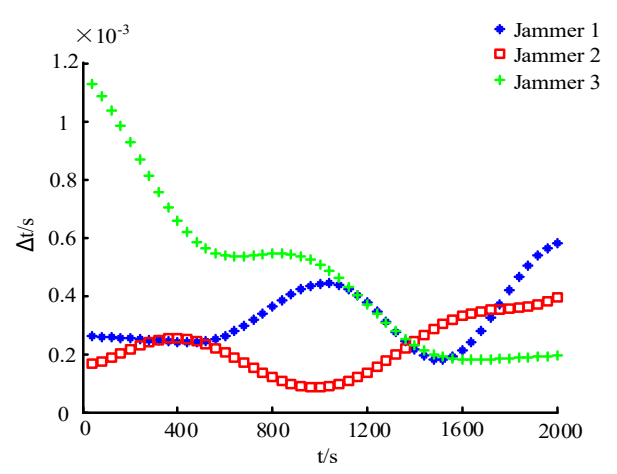

Fig.9.Jammers' Signal Delay Amount

\section{Conclusion}

This article is based on the traditional track deception jamming technology, considers the situation that the jammers needs to adjust the route, expands the degree of freedom of the jammers' route, and gives the movement model and the solving process of the jammers under the flexible route penetration. Then the parameters of jammers were obtained through simulation. This technology not only can achieve track deception, but also can control the jammer route more flexibly. It can better cooperate with suppressing jamming when it comes to radar network penetration, and provides a reference for deception and suppression of the organic combination of interference.

\section{REFERENCES}

1. FAN zhenyu, WANG Lei, SU Jianchun. Technology of track deception for cooperative control of multiple Electronic Combat Air Vehicles [J]. INFORMATION AND ELECTRONIC ENGINEERING,2010,8(3):265-268.

2. LI Fei,ZHOU Zhongliang,GOU Xinyu, et al.Technology for penetrating radar net based on multiple combat air vehicles cooperation track deception. [J].Systems Engineering and Electronics, 2013,35(11):2309-2313.

3. YANG Zhong, WANG Guohong, SUN Dianxing. Collborative Programming Technic of Radar Network in Presence of Phantom Track Deception [J]. Command Control \& Simulation,2015,37(6):4549.

4. LI Xiaobo, SUN Lin, ZHOU Qingsong.Strategy for Track Deception Jamming Against Radar Network Using Cooperative Autonomous Vehicle Teams $[\mathrm{J}]$. MODERN DEFENCE TECHNOLOGY,2016,44(6):43-49.

5. GUO Shufen, YU Guowen, XIONG Xin.Research on method of track deception jamming based on cooperative control of UAVs [J]. Journal of Air Force Early Warning Academy,2018,32(1):44-54.

6. LIU Xiang, LI Dongsheng. The deviation compensation for Phantom Tracks Jamming against 
distributed Radar Network [J]. Systems Engineering and Electronics,2018,40.

7. LI Xin,WANG Chunyang. Track deception jamming and its countermeasures Research status and development [J]. Cruise Missile,2013(8):64-67.

8. WU Yuqing. AIMING At the Ntted Radar Electronic Interfrence technology research [D]. School of Electronic Engineering ,2013.60-65.

9. MA Yatao. Research on Deception Jamming against the Network System of Radars [D]. Xi'an University of Electronic Science and Technology,2013.37-45. 